\title{
Prefiltering-Based Interference Suppression for Time-Hopping Multiuser UWB Communications over MISO Channel
}

\author{
Wei-Chiang Wu \\ Department of Electrical Engineering, Da-Yeh University, 168 University Rd., Dacun, Changhua 51591, Taiwan \\ Correspondence should be addressed to Wei-Chiang Wu, nash.mcquire@msa.hinet.net \\ Received 30 January 2009; Revised 17 April 2009; Accepted 10 June 2009 \\ Recommended by Jonathon Chambers
}

\begin{abstract}
This paper proposes a prefiltering-based scheme for pulsed ultra-wideband (UWB) system by shifting the signal processing needs from the receiver at the radio terminal (RT) to the transmitter at the fixed access point (AP) where power and computational resources are plentiful. We exploit antenna array in the transmitter of AP and take advantage of the spatial and temporal diversities to mitigate the multiuser interference (MUI) as well as preequalize the channel impulse response (CIR) of a timehopping $(\mathrm{TH})$ multiple access UWB communication system. Three prefiltering schemes are developed to meet different criteria. A simple correlation receiver is proposed at the RT to combine the desired signal stemmed from all the transmitting antennas. The performances under different scenarios are extensively evaluated over multiple-input single-output (MISO) channels.
\end{abstract}

Copyright (C) 2009 Wei-Chiang Wu. This is an open access article distributed under the Creative Commons Attribution License, which permits unrestricted use, distribution, and reproduction in any medium, provided the original work is properly cited.

\section{Introduction}

Recently, a lot of attention was paid to UWB impulse radio systems since it is a promising technique for low-complexity low-power short-range indoor wireless communications [15]. When such transmissions are applied in multiple access system, time-hopping ( $\mathrm{TH})$ spreading codes are a plausible choice to separate different users [6-8]. Modulation of TH impulse radio is accomplished by assigning user-specific pattern of time shifting of pulses.

The time-reversal- (TR-) based UWB scheme has been extensively investigated recently [9-13]. Attractive features of TR signal processing include the following.

(1) It makes full use of the energy from all the resolvable paths: it can create space and time focalization at a specific point where signals are coherently added [9, $12]$.

(2) Channel estimation in UWB system is generally a difficult task. Most UWB networks have APs, and the TR-based UWB technique shifts the sophisticated channel estimation burden from the receivers of radio terminal (RT) to the AP. This is also referred to as the "prerake" diversity combining scheme [14].
(3) Quite a few data-aided and blind timing acquisition schemes have been proposed [15-18] for UWB transmission through dense multipath channels. Synchronization in TR UWB scheme is extremely simplified since the peak is automatically created and aligned of the received signal at specific time slot.

Most past works of TR UWB scheme focus on the issue of single-user transmission and detection. The topic of multiuser TR UWB scheme has been analyzed in [19]. With different approach, we employ TH codes and address the applicability of zero-forcing (ZF) and least squares (LS) techniques to further improve system performance. The communication system considered in this paper consists of $M$ transmitting antennas at the AP, $K$ single-antenna RT, which indicates $K$ individual MISO channels. Signal separation is accomplished by

(1) user-specific TH codes that are designed as "orthogonal" as possible, that is, locate each user's pulse train in nonoverlapping time slots.

(2) user-specific CIR that is determined by each user's spatial location.

In this paper, we propose three prefiltering schemes, where a set of $M$ prefilters are designated to each user 
at the transmitter of the AP. The prefilters of the first scheme are derived to meet the ZF criterion such that MUI is completely removed at the receiver front end of RT. Thereby, a simple single-user correlator can be employed at RT receiver to maximize output signal-to-noise ratio (SNR). When the degrees of freedom are insufficient for complete MUI suppression, an LS-based scheme is also proposed to mitigate MUI. The third scheme is composed of a set of TR matchedfilters (MFs) at the transmitter that correlate to the MISO CIR. Since the TR MF technique with application in the MISO UWB system has excellent spatial-temporal focusing capability, the energy of the received signal tends to concentrate on some controllable time slots. This enables us to implement a simple correlation receiver to extract the energy at these time slots where peak occur.

The remainder of this paper is organized as follows. In Section 2, we formulate the signal and channel models of the time-hopping UWB multiple access communication system over frequency-selective fading channel. Section 3 highlights the rationale of the prefiltering-based multiuser UWB MISO system, where three prefiltering schemes are proposed for signal transmission and detection. Simulation results are presented and analyzed in Section 4. Concluding remarks are finally made in Section 5.

Notation. The boldface letters represent vector or matrix. $\mathbf{A}(i, j)$ denotes the element of $i$ th row and $j$ th column of matrix $\mathbf{A}, \mathbf{x}(l)$ denotes the $l$ th element of vector $\mathbf{x}$, and []$^{T}$ and []$^{H}$ stand for transpose and complex transpose of a matrix or vector, respectively. We will use $E\{\}$ for expectation (ensemble average), || || for vector norm, and := for "is defined as." Also, "*” indicates the linear convolution operation, $\mathbf{I}_{M}$ denotes an identity matrix with size $M$, and $\mathbf{0}_{M}, \mathbf{1}_{M}$ are $M \times 1$ vectors with all elements being 0 and 1 , respectively. Finally, $\delta(\cdot)$ is the dirac delta function.

\section{Signal and Channel Models}

2.1. Signal Model. In UWB impulse radios, every information symbol (bit) is conveyed by $N_{f}$ data modulated ultrashort pulses over $N_{f}$ frames. There is only one pulse in each frame, and the frame duration is $T_{f}$. The pulse waveform, $p(t)$, is referred to as a monocycle [1] with ultrashort duration $T_{c}$ at the nanosecond scale. The energy of $p(t)$ is normalized within $T_{c}$ to unity, so that $\int_{0}^{T_{c}}|p(t)|^{2} d t=$ 1. Note that $T_{f}$ is usually a hundred to a thousand times of chip duration, $T_{c}$, which accounts for very low duty cycle. When multiple users are simultaneously transmitted and received, signal separation can be accomplished with user-specific pseudorandom TH codes, which shift the pulse position in every frame. The binary (antipodal) PAM scheme is considered, thus we may establish the signal model designated for the $k$ th $\mathrm{RT}$ as

$$
\begin{aligned}
s_{k}(t) & =\sum_{i} a_{k} d_{k}(i) c_{k}(t) \\
& =\sum_{i} a_{k} d_{k}(i) \sum_{j=0}^{N_{f}-1} p\left(t-i N_{f} T_{f}-j T_{f}-c_{j}^{k} T_{c}\right),
\end{aligned}
$$

where $t$ is the clock time of the transmitter, and $i$ is the bit index. $a_{k}$ is the amplitude. Binary information bit $d_{k}(i)$ takes on the value \pm 1 with equal probability. $c_{k}(t):=$ $\sum_{j=0}^{N_{f}-1} p\left(t-i N_{f} T_{f}-j T_{f}-c_{j}^{k} T_{c}\right)$ represents the specific waveform assigned for the $k$ th RT. Denoting $T_{b}$ as the bit duration, then $T_{b}=N_{f} T_{f}$. Suppose each frame is composed of $N_{c}$ time slots each with duration $T_{c}$, thus, $T_{f}=N_{c} T_{c}$. User separation is accomplished by user-specific pseudo-random TH code. $\left\{c_{j}^{k}\right\}_{j=0, \ldots, N_{f}-1}$ accounts for the $k$ th user's TH code with period $N_{f}$. Thereby $c_{j}^{k} T_{c}$ is the time-shift of the pulse position imposed by the $\mathrm{TH}$ sequence employed for multiple access. $c_{j}^{k} T_{c} \leq T_{f}$, or equivalently, $0 \leq c_{j}^{k} \leq N_{c}-1$. Note that to avoid the presence of intersymbol interference (ISI), we let the last frame for each user being empty (without pulse). This is equivalent to adding a guard time $T_{f}$ at the end of each bit. Specifically, $T_{f}$, which is up to our disposal, should be longer than the sum of delay spread (maximum dispersion), $T_{d}$, of the CIR and the prefilter length. Based on the signal model of (1), the transmitted bit energy for the $k$ th user can be calculated as $E_{b, k}=\left(N_{f}-1\right) a_{k}^{2}$. $E_{b, k}$ is chosen to meet the FCC regulated power level such that the UWB technology is allowed to overlay already available services.

2.2. Channel Model. Most of the envisioned commercial UWB applications will be indoor communications. The CIR as observed in the measurement of indoor environment can be expressed in general as [20]

$$
h(t)=\sum_{n=0}^{N} \sum_{l=0}^{L} \alpha_{n, l} \exp \left(j \phi_{n, l}\right) \delta\left(t-T_{n}-\tau_{n, l}\right),
$$

where $\alpha_{n, l}$ and $\phi_{n, l}$ are the gain (attenuation) and phase of the $l$ th multipath component (MPC) of the $n$th cluster, respectively. $T_{n}+\tau_{n, l}\left(\tau_{n, 0}=0\right)$ denotes the arrival time of the $l$ th MPC of the $n$th cluster. Cluster arrivals and the subsequent arrivals within each cluster are modeled as Possion distribution with different rates. As described in [20], for some environments, most notably the industrial (CM9) and indoor office (CM4), "dense" arrivals of MPC were observed, that is, each resolvable delay bin contains significant energy. In these cases, the concept of ray arrival rates loses its meaning, and a realization of the impulse response- (IR-) based on a tapped delay line model with regular tap spacings is to be used, that is, a single cluster $(N=1)$, so that $\tau_{1, l}=\tau_{l}=l \Delta \tau$, where $\Delta \tau=T_{c}$ is the spacing of the delay taps. Moreover, the phase term, $\phi_{n, l}$, is also constrained to take values 0 or $\pi$ with equal probability to account for the random pulse inversion due to reflection [21], so that $\exp \left(j \phi_{n, l}\right)= \pm 1$ with equal probability. This yields a real-valued channel model. Considering the above factors, the CIR of (2) can be reformulated as

$$
h(t)=\sum_{l=0}^{L} \alpha_{l} \delta\left(t-l T_{c}\right)
$$

where we model the multipath channel as a tapped-delay line with $(L+1)$ taps. $\alpha_{l}$ denotes the tap weight of the $l$ th resolvable path. Note that in writing (3), we have implicitly 


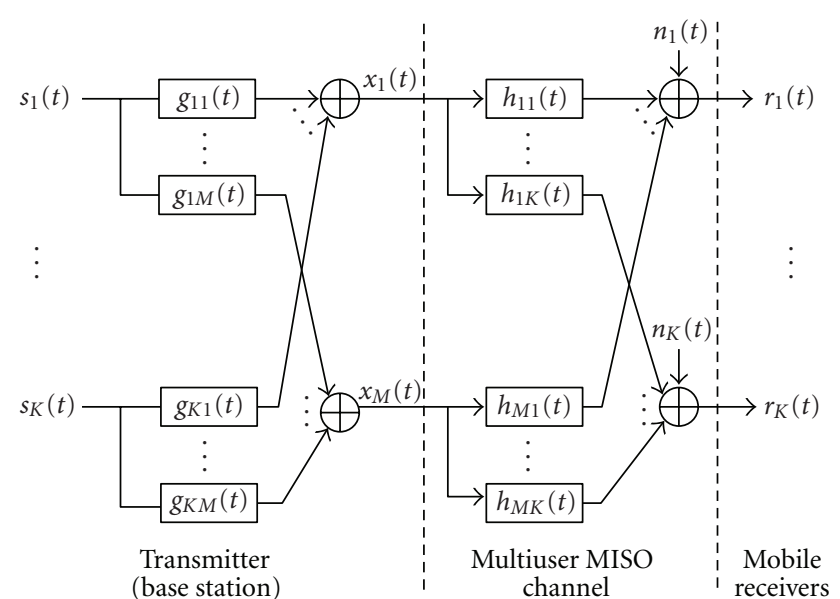

(a)

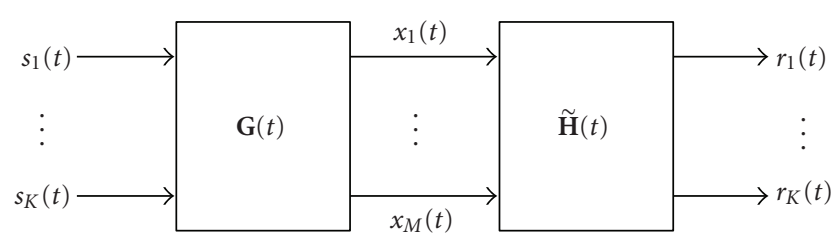

(b)

FIGURE 1: Schematic block diagram of a prefiltering-based MISO UWB communication system.

assumed that maximum time dispersion is $L T_{c}$. The channel fading coefficient $\alpha_{l}$ can be modeled as [22]

$$
\alpha_{l}=b_{l} \xi_{l}
$$

where $b_{l}=\exp \left(j \phi_{n, l}\right)$ is equiprobable to take on the value \pm 1 . $\xi_{l}=\left|\alpha_{l}\right|$ is the log-normal fading magnitude term. The average power of $\alpha_{l}$ is represented by $E\left\{\left|\alpha_{l}\right|^{2}\right\}=$ $\Omega_{0} \exp (-\rho l) . \Omega_{0}$ is a scalar for normalizing the power contained in resolvable paths, and $\rho$ is the power decay factor. To simplify the analysis, we assume that the channel parameters are quasistatic (slowly fading) such that they are essentially constant over observation interval.

\section{Design of Transmitters and Receivers in Prefiltered UWB MISO System}

3.1. General Prefiltered UWB MISO System. As shown in Figure 1 of the considered structure, there are $M$ transmitting antennas equipped at the AP, and each RT has single antenna. Let $h_{m k}(t)=\sum_{l=0}^{L} \alpha_{m k, l} \delta\left(t-l T_{c}\right)$ denote the CIR between the $m$ th transmitting antenna and the $k$ th RT, where $\alpha_{m k, l}$ represents the fading coefficient of the $l$ th path. In the proposed prefiltering scheme, a set of $M K$ finite impulse response (FIR) prefilters with IRs $g_{k m}(t)=\sum_{p=0}^{P-1} \beta_{k m, p} \delta\left(t-p T_{c}\right)$ are inserted, respectively, between $s_{k}(t)$ and the $m$ th transmitting antenna. All users are synchronously transmitted from the AP to all the RTs. Thereby, the transmitted waveform at the $m$ th antenna is

$$
x_{m}(t)=\sum_{k=1}^{K} s_{k}(t) * g_{k m}(t) ; \quad m=1, \ldots, M,
$$

where $K$ is the number of RTs. Upon defining $\mathbf{x}(t):=$ $\left[\begin{array}{llll}x_{1}(t) & x_{2}(t) & \cdots & x_{M}(t)\end{array}\right]^{T}, \mathbf{s}(t):=\left[\begin{array}{lllll}s_{1}(t) & s_{2}(t) & \cdots & s_{K}(t)\end{array}\right]^{T}$ and the $K \times M$ prefiltering matrix

$$
\mathbf{G}(t):=\left[\begin{array}{cccc}
g_{11}(t) & g_{12}(t) & \cdots & g_{1 M}(t) \\
g_{21}(t) & g_{22}(t) & \cdots & g_{2 M}(t) \\
\vdots & \ddots & \ddots & \vdots \\
g_{K 1}(t) & g_{K 2}(t) & \cdots & g_{K M}(t)
\end{array}\right] .
$$

We may reexpress (5) as a compact form:

$$
\mathbf{x}(t)=\mathbf{G}^{T}(t) * \mathbf{s}(t) .
$$

The channel between the AP and arbitrary RT can be regarded as a MISO system. Hence, the received signal at the $k$ th RT can be formulated as

$$
\begin{aligned}
r_{k}(t)= & \sum_{m=1}^{M} x_{m}(t) * h_{m k}(t)+n_{k}(t) \\
= & \sum_{m=1}^{M}\left(\sum_{j=1}^{K} s_{j}(t) * g_{j m}(t)\right) * h_{m k}(t) \\
& +n_{k}(t) ; \quad k=1, \ldots, K \\
= & \sum_{j=1}^{K} s_{j}(t) * \sum_{m=1}^{M}\left(g_{j m}(t) * h_{m k}(t)\right)+n_{k}(t),
\end{aligned}
$$

where $n_{k}(t)$ is assumed to be zero-mean AWGN noise process with variance $\sigma^{2}$ (assume independent of $k$ for simplicity). Similarly, let $\mathbf{r}(t):=\left[\begin{array}{llll}r_{1}(t) & r_{2}(t) & \cdots & r_{K}(t)\end{array}\right]^{T}, \mathbf{n}(t):=$ $\left[\begin{array}{llll}n_{1}(t) & n_{2}(t) & \cdots & n_{K}(t)\end{array}\right]^{T}$ and the $M \times K$ MISO multiuser channel matrix

$$
\tilde{\mathbf{H}}(t):=\left[\begin{array}{cccc}
h_{11}(t) & h_{12}(t) & \cdots & h_{1 K}(t) \\
h_{21}(t) & h_{22}(t) & \cdots & h_{2 K}(t) \\
\vdots & \ddots & \ddots & \vdots \\
h_{M 1}(t) & h_{M 2}(t) & \cdots & h_{M K}(t)
\end{array}\right] .
$$

We may reformulate (8) into a more convenient form:

$$
\begin{aligned}
\mathbf{r}(t) & =\widetilde{\mathbf{H}}^{T}(t) * \mathbf{x}(t)+\mathbf{n}(t) \\
& =\widetilde{\mathbf{H}}^{T}(t) * \mathbf{G}^{T}(t) * \mathbf{s}(t)+\mathbf{n}(t) \\
& =[\mathbf{G}(t) * \tilde{\mathbf{H}}(t)]^{T} * \mathbf{s}(t)+\mathbf{n}(t) \\
& =\mathbf{H}_{\mathrm{eff}}^{T}(t) * \mathbf{s}(t)+\mathbf{n}(t),
\end{aligned}
$$

where $\mathbf{H}_{\text {eff }}(t):=\mathbf{G}(t) * \tilde{\mathbf{H}}(t)$ represents the "effective" FIR channel matrix with size $K \times K$. 


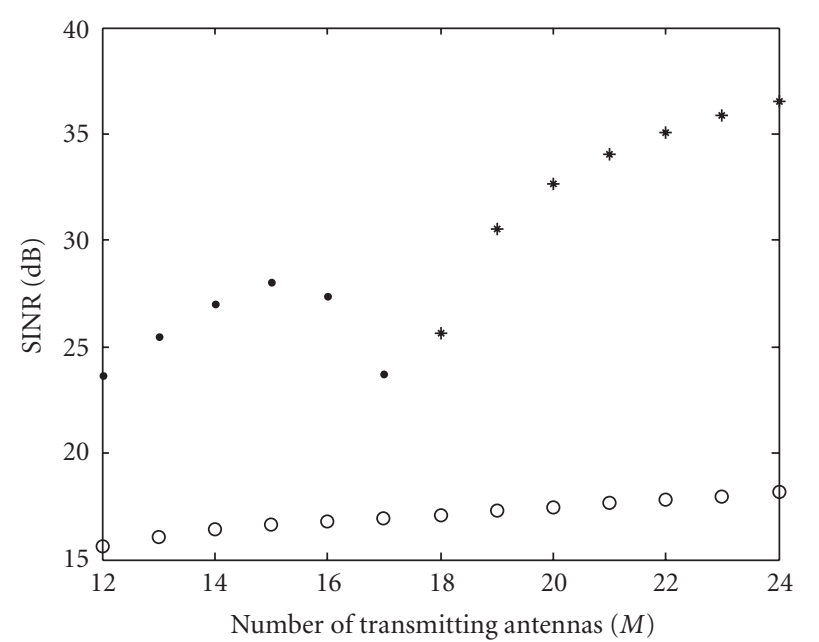

- LS scheme

* ZF scheme

○ TR-MF scheme

FIGURE 2: SINR performance with respect to the number of transmitting antennas.

3.2. Zero-Forcing- (ZF-) Based Scheme. To completely remove MUI, the $M K$ prefilters should be designed to satisfy the following ZF criteria:

$$
\begin{aligned}
& \sum_{m=1}^{M}\left(g_{j m}(t) * h_{m k}(t)\right) \\
& \quad=\left\{\begin{array}{ll}
0, & j \neq k, \\
\eta_{k} \delta\left(t-L T_{c}\right), & j=k,
\end{array} \quad \forall j, k=1, \ldots, K,\right.
\end{aligned}
$$

where $L T_{c}$ is the delay introduced to accommodate the multipath effect. $\eta_{k}$ accounts for the power normalization factor designated for each RT so that the transmitter bit energy remains constant independent of the number of transmit antennas. In other words, ZF-based prefilters attempt to design $\mathbf{G}(t)$ such that $\mathbf{H}_{\mathrm{eff}}(t)$ reduces to a diagonal matrix

$$
\mathbf{H}_{\mathrm{eff}}^{(\mathrm{ZF})}(t):=\mathbf{G}^{(\mathrm{ZF})}(t) * \tilde{\mathbf{H}}(t)=\left[\begin{array}{ccc}
\eta_{1} & \cdots & 0 \\
\vdots & \ddots & \vdots \\
0 & \cdots & \eta_{K}
\end{array}\right] \delta\left(t-L T_{c}\right)
$$

Denoting the discrete-time version $h_{m k}(t)$ as a $(L+1)$ vector $\mathbf{h}_{m k}:=\left[\begin{array}{lllll}\alpha_{m k, 0} & \alpha_{m k, 1} & \cdots & \alpha_{m k, L}\end{array}\right]^{T}, g_{j m}(t)=$ $\sum_{p=0}^{P-1} \beta_{j m, p} \delta\left(t-p T_{c}\right)$ as a $P$ vector $\mathbf{g}_{j m}:=$ $\left[\begin{array}{llll}\beta_{j m, 0} & \beta_{j m, 1} & \cdots & \beta_{j m, P-1}\end{array}\right]^{T}$ (Here, we assume the order of the FIR prefilters being $P$ ), respectively, then the discretetime counterparts of $g_{j m}(t) * h_{m k}(t)$ can be obtained as

$$
\mathbf{H}_{m k} \mathbf{g}_{j m}=\left[\begin{array}{ccccc}
\alpha_{m k, 0} & 0 & \cdots & \cdots & 0 \\
\vdots & \alpha_{m k, 0} & \ddots & \ddots & \vdots \\
\alpha_{m k, L} & \vdots & \ddots & \ddots & \vdots \\
0 & \alpha_{m k, L} & \ddots & \ddots & 0 \\
\vdots & 0 & \ddots & \ddots & \alpha_{m k, 0} \\
\vdots & \vdots & \ddots & \ddots & \vdots \\
0 & 0 & \cdots & 0 & \alpha_{m k, L}
\end{array}\right]\left[\begin{array}{c}
\beta_{j m, 0} \\
\beta_{j m, 1} \\
\vdots \\
\beta_{j m, P-1}
\end{array}\right],
$$

where $\mathbf{H}_{m k}$ is a $(L+P) \times P$ matrix as defined in (13). Using (13), we may convert (11) into

$$
\mathbf{H}_{k} \mathbf{g}_{j}= \begin{cases}\mathbf{0}_{L+P}, & j \neq k, \\ \eta_{k} \mathbf{e}_{L}, & j=k,\end{cases}
$$

where $\mathbf{e}_{L}$ denotes the $L$ th column vector of $\mathbf{I}_{(L+P)} \cdot \mathbf{H}_{k}:=$ $\left[\begin{array}{llll}\mathbf{H}_{1 k} & \mathbf{H}_{2 k} & \cdots & \mathbf{H}_{M k}\end{array}\right]$ is a $(L+P) \times M P$ matrix, and $\mathbf{g}_{j}:=$ $\left[\begin{array}{llll}\mathbf{g}_{j 1}^{T} & \mathbf{g}_{j 2}^{T} & \cdots & \mathbf{g}_{j M}^{T}\end{array}\right]^{T}$ is a $M P$ vector that incorporates the space-time IR of the $j$ th user's prefilters. Upon defining the $K(L+P) \times M P$ matrix $\mathbf{H}:=\left[\begin{array}{llll}\mathbf{H}_{1}^{T} & \mathbf{H}_{2}^{T} & \ldots & \mathbf{H}_{K}^{T}\end{array}\right]^{T}$ and the $K(L+P)$ vector, $\mathbf{e}_{L}^{(k)}:=\left[\begin{array}{lllll}\mathbf{0}_{L+P}^{T} & \cdots & \mathbf{e}_{L}^{T} & \cdots & \mathbf{0}_{L+P}^{T}\end{array}\right]^{T}$, we may reexpress (14) as

$$
\mathbf{H g}_{k}=\eta_{k} \mathbf{e}_{L}^{(k)}
$$

where $\mathbf{e}_{L}^{(k)}$ denotes the $(k(L+P)-P)$ th column vector of $\mathbf{I}_{K(L+P)}$.

If $K(L+P) \leq M P$, which is up to our disposal (can be achieved by increasing $M$ and/or $P$ ), we have infinitely many solutions since (15) is indeed an underdetermined system. The general solution includes a quiescent solution and a homogeneous solution that is chosen from the null (kernel) space of $\mathbf{H}$ :

$$
\mathbf{g}_{k}^{(\mathrm{ZF})}=\eta_{k} \mathbf{H}^{T}\left(\mathbf{H H}^{T}\right)^{-1} \mathbf{e}_{L}^{(k)}+\mathbf{u} ; \quad k=1, \ldots, K,
$$

where $\mathbf{H u}=\mathbf{0}$. It should be noted that $\mathbf{u}$ can be regarded as the surplus part of $\mathbf{g}_{k}$ since it is useless for MUI suppression but only wastes transmission power. Thus, we let $\mathbf{u}=\mathbf{0}$ to minimize power consumption. Moreover, to guarantee the transmitted bit energy of the $k$ th user to be $E_{b, k}=\left(N_{f}-\right.$ 1) $a_{k}^{2}$ independent of the prefilters and number of antennas, we choose $\eta_{k}$ such that $\left\|\mathbf{g}_{k}\right\|=1$. It follows from (16) (after removing $\mathbf{u}$ )

$$
\begin{aligned}
\left\|\mathbf{g}_{k}\right\|^{2} & =\eta_{k}^{2} \mathbf{e}_{L}^{(k)^{T}}\left[\mathbf{H} \mathbf{H}^{T}\right]^{-1} \mathbf{e}_{L}^{(k)} \\
& =\eta_{k}^{2}\left[\mathbf{H} \mathbf{H}^{T}\right]^{-1}(k(L+P)-P, k(L+P)-P)
\end{aligned}
$$


Hence, $\eta_{k}$ is chosen as

$$
\eta_{k}^{(\mathrm{ZF})}=\frac{1}{\sqrt{\left[\mathbf{H H}^{T}\right]^{-1}(k(L+P)-P, k(L+P)-P)}} .
$$

At the front end of each RT, chip-matched filtering (CMF) followed by chip-rate sampling, the discrete-time counterpart of $r_{k}(t)$, can be obtained as

$$
r_{k}(n)=s_{k}(n) * \eta_{k} \delta(n-L)+n_{k}(n) ; \quad k=1, \ldots, K,
$$

where the interference has been removed by ZF prefiltering. After bit-by-bit stacking, we arrive at a sequence of $N_{c} N_{f}$ vectors. The samples of CMF output within the $i$ th bit interval at the $k$ th $\mathrm{RT}$ are

$$
\mathbf{r}_{k}(i)=\eta_{k} a_{k} d_{k}(i) \mathbf{c}_{k, L}+\mathbf{n}_{k}(i)
$$

where $\mathbf{c}_{k, L}$ stands for an $L$-chips delayed version of the $k$ th user's TH code vector $\mathbf{c}_{k}$. The delay results from the criterion of (11). Since the MUI is completely removed, a simple correlation receiver can be employed that maximizes the averaged output SNR. The output signal (denoted as $z_{k}^{(\mathrm{ZF})}$ ), averaged SNR (denoted as $\gamma_{k}^{(\mathrm{ZF})}$ ), and bit-error-rate (BER) (denoted as $P e_{k}^{(\mathrm{ZF})}$ ) can be obtained in order:

$$
\begin{aligned}
z_{k}^{(\mathrm{ZF})}(i) & =\mathbf{c}_{k, L}^{T} \mathbf{r}_{k}(i)=\eta_{k} a_{k} d_{k}(i)\left\|\mathbf{c}_{k, L}\right\|^{2}+\mathbf{c}_{k, L}^{T} \mathbf{n}_{k}(i) \\
& =\eta_{k}\left(N_{f}-1\right) a_{k} d_{k}(i)+\mathbf{c}_{k, L}^{T} \mathbf{n}_{k}(i) \\
\gamma_{k}^{(Z F)} & =\frac{\eta_{k}^{2} a_{k}^{2}\left(N_{f}-1\right)^{2}}{\sigma^{2}\left(N_{f}-1\right)}=\frac{\eta_{k}^{2} a_{k}^{2}\left(N_{f}-1\right)}{\sigma^{2}} \\
P e_{k}^{(Z F)} & =Q\left(\frac{\eta_{k} a_{k} \sqrt{\left(N_{f}-1\right)}}{\sigma}\right)
\end{aligned}
$$

where $Q(x):=1 / \sqrt{2 \pi} \int_{x}^{\infty} \exp \left(-\left(v^{2} / 2\right)\right) d v$ is a monotonically decreasing function with respect to $x$.

If on the other hand, $K(L+P)>M P$, or equivalently $K>M P /(L+P)$, the $\mathrm{ZF}$ criteria are inapplicable since there is insufficient degrees of freedom to suppress the interference. In other words, (15) becomes an overdetermined system. It is generally impossible to obtain exact solution. By minimizing the least-squares (minimum distance) criterion [23], we can obtain the $k$ th user's prefilter as

$$
\mathbf{g}_{k}^{(\mathrm{LS})}=\eta_{k}\left(\mathbf{H}^{T} \mathbf{H}\right)^{-1} \mathbf{H}^{T} \mathbf{e}_{L}^{(k)} .
$$

Similar to the derivation in (17) and (18), the power normalization factor can be obtained as

$$
\begin{array}{r}
\eta_{k}^{(\mathrm{LS})}=\frac{1}{\sqrt{\left[\mathbf{H}\left(\mathbf{H}^{T} \mathbf{H}\right)^{-2} \mathbf{H}^{T}\right](k(L+P)-P, k(L+P)-P)}}, \\
k=1, \ldots, K .
\end{array}
$$

Note that the LS solution can only "approximate" the ZF criteria. Therefore, the received signal at the $k$ th RT should contain residual MUI:

$$
\begin{aligned}
\mathbf{r}_{k}(i) & =\sum_{j=1}^{K} a_{j} d_{j}(i) \mathbf{c}_{j} * \mathbf{H}_{k} \mathbf{g}_{j}+\mathbf{n}_{k}(i) \\
& =\sum_{j=1}^{K} \eta_{j} a_{j} d_{j}(i) \mathbf{c}_{j k}+\mathbf{n}_{k}(i),
\end{aligned}
$$

where $\mathbf{c}_{j k}:=\mathbf{c}_{j} * \mathbf{H}_{k} \mathbf{g}_{j}$. Applying the same correlator as ZF scheme, we can obtain the output averaged signal-tointerference-plus-noise ratio (SINR) as

$$
\gamma_{k}^{(\mathrm{LS})}=\frac{\eta_{k}^{2} a_{k}^{2} \rho_{k}^{2}}{\sum_{j=1, j \neq k}^{K} a_{j}^{2} \eta_{j}^{2} \rho_{j}^{2}+\sigma^{2}\left(N_{f}-1\right)},
$$

where $\rho_{j}:=\mathbf{c}_{k, L}^{T} \mathbf{c}_{j k}$. With some manipulations, we can deduce the BER for the LS-based scheme:

$P E_{k}^{(\mathrm{LS})}$

$$
=2^{1-K} \sum_{\substack{d_{1} \cdots d_{K} \in\{-1,+1\} \\ d_{k}=1}} Q\left(\frac{\eta_{k} a_{k} \rho_{k}+\sum_{j=1, j \neq k}^{K} \eta_{j} a_{j} \rho_{j} d_{j}}{\sigma \sqrt{N_{f}-1}}\right),
$$

3.3. TR-MF-Based Scheme. In this scheme, a set of TR MFs with IRs $g_{k m}(t)=\eta_{k m} h_{m k}\left(T_{d}-t\right) ; k=1, \ldots, K, m=1, \ldots, M$ are placed at the transmitter as the prefilters, where $T_{d}$ denotes the delay spread (maximum dispersion) of the CIR. In the considered model, $T_{d}=L T_{c}$, thus we may rewrite the IR of the TR MF as

$$
\begin{array}{r}
g_{k m}(t)=\eta_{k m} h_{m k}\left(T_{d}-t\right)=\eta_{k m} \sum_{l=0}^{L} \alpha_{m k, L-l} \delta\left(t-l T_{c}\right), \\
m=1, \ldots, M, k=1, \ldots, K .
\end{array}
$$

To guarantee that the energy per transmitted bit remains $E_{b, k}=\left(N_{f}-1\right) a_{k}^{2}$ independent of the prefilters and number of antennas, it is easy to deduce that $\eta_{k m}$ should be chosen as

$$
\eta_{k m}=\frac{1}{\sqrt{M \sum_{l=0}^{L} \alpha_{m k, L-l}^{2}}}=\frac{1}{\sqrt{M}\left\|\mathbf{h}_{m k}\right\|} .
$$

Apparently, the order of the prefilters in the TR-MF scheme is the same as the MISO CIR. Let $\hat{h}_{m k}\left(T_{d}-t\right):=\eta_{k m} h_{m k}\left(T_{d}-t\right)$, then the $K \times M$ prefiltering matrix for the TR MF scheme can be expressed as

$$
\mathbf{G}^{(\mathrm{MF})}(t):=\left[\begin{array}{cccc}
\hat{h}_{11}\left(T_{d}-t\right) & \hat{h}_{12}\left(T_{d}-t\right) & \cdots & \hat{h}_{1 M}\left(T_{d}-t\right) \\
\hat{h}_{21}\left(T_{d}-t\right) & \hat{h}_{22}\left(T_{d}-t\right) & \cdots & \hat{h}_{2 M}\left(T_{d}-t\right) \\
\vdots & \ddots & \ddots & \vdots \\
\hat{h}_{K 1}\left(T_{d}-t\right) & \hat{h}_{K 2}\left(T_{d}-t\right) & \cdots & \hat{h}_{K M}\left(T_{d}-t\right)
\end{array}\right] .
$$




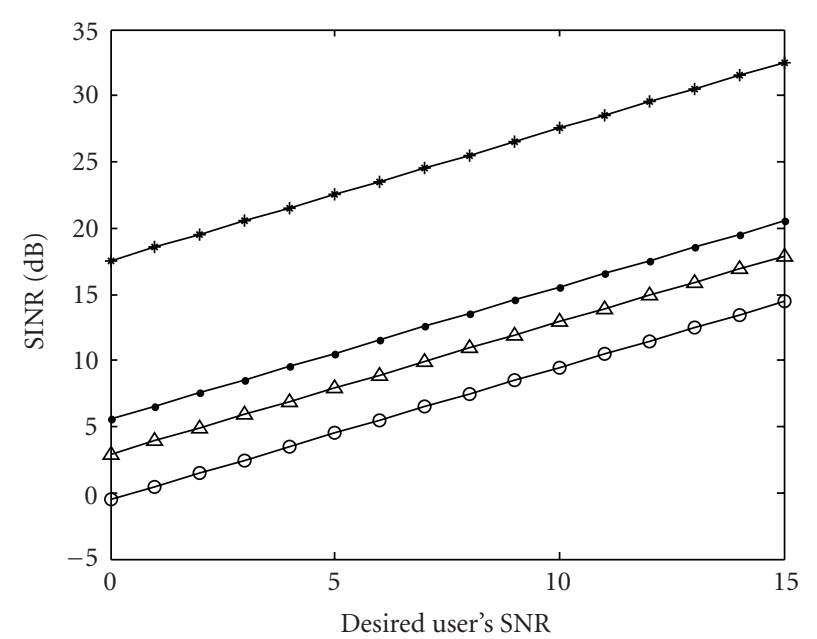

$\rightarrow$ LS scheme $(M=10) \quad \rightarrow \quad$ ZF scheme $(M=20)$ $\multimap$ TR-MF scheme $(M=10) \triangle$ TR-MF scheme $(M=20)$

(a) SINR performance with respect to the desired user's SNR.

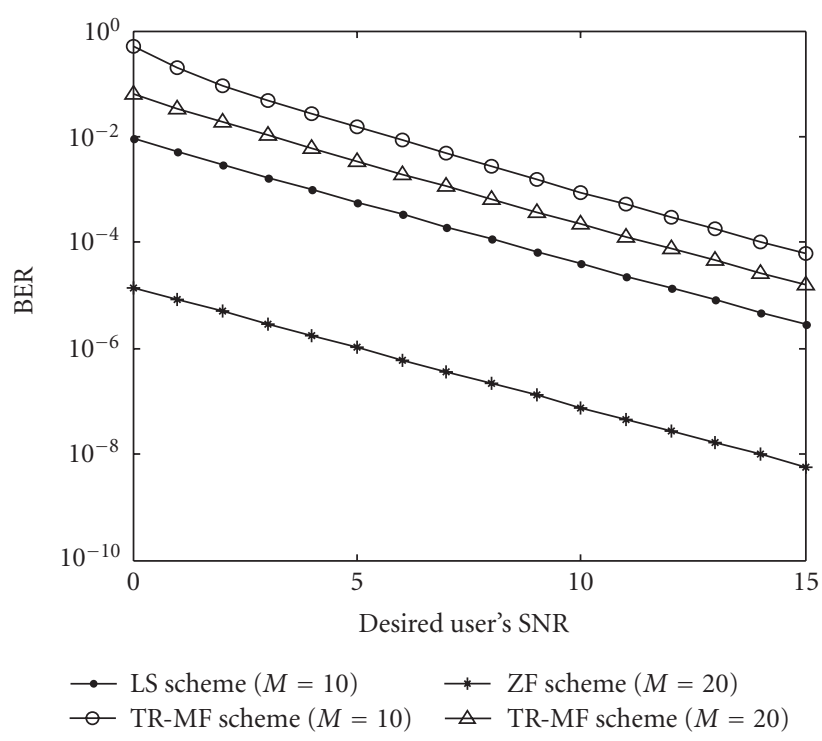

(b) BER performance with respect to the desired user's SNR.

FIGURE 3: System performance with respect to the desired user's SNR.

The transmitted waveform at the $m$ th antenna is

$$
x_{m}(t)=\sum_{k=1}^{K} s_{k}(t) * \hat{h}_{m k}\left(T_{d}-t\right), \quad m=1, \ldots, M .
$$

Substituting (30) into (8), the received signal at the $k$ th RT can be obtained as

$$
\begin{aligned}
r_{k}(t)= & \sum_{m=1}^{M} x_{m}(t) * h_{m k}(t)+n_{k}(t) \\
= & \sum_{m=1}^{M}\left\{\sum_{j=1}^{K} s_{j}(t) * \hat{h}_{m j}\left(T_{d}-t\right)\right\} * h_{m k}(t)+n_{k}(t) \\
= & \sum_{m=1}^{M}\left\{s_{k}(t) * \hat{h}_{m k}\left(T_{d}-t\right) * h_{m k}(t)\right\} \\
& +\sum_{m=1}^{M} \sum_{\substack{j=1 \\
j \neq k}}^{K}\left\{s_{j}(t) * \hat{h}_{m j}\left(T_{d}-t\right) * h_{m k}(t)\right\}+n_{k}(t) \\
= & \sum_{m=1}^{M}\left\{s_{k}(t) * R_{m k}(t)\right\} \\
& +\sum_{m=1}^{M} \sum_{\substack{j=1 \\
j \neq k}}^{K}\left\{s_{j}(t) * R_{m j, m k}(t)\right\}+n_{k}(t) \\
= & s_{k}(t) * R_{k}(t)+\sum_{\substack{j=1 \\
j \neq k}}^{K}\left\{s_{j}(t) * R_{j, k}(t)\right\}+n_{k}(t)
\end{aligned}
$$

where $R_{m k}(t):=\hat{h}_{m k}\left(T_{d}-t\right) * h_{m k}(t)$ is the autocorrelation function of $h_{m k}(t), R_{m j, m k}(t):=\hat{h}_{m j}\left(T_{d}-t\right) * h_{m k}(t)$ accounts for the cross-correlation function between $h_{m k}(t)$ and $h_{m j}(t)$. $R_{k}(t):=\sum_{m=1}^{M} R_{m k}(t), R_{j, k}(t):=\sum_{m=1}^{M} R_{m j, m k}(t)$. In what follows, the "effective" FIR channel matrix for the TR MF scheme yields

$$
\begin{aligned}
\mathbf{H}_{\mathrm{eff}}^{(\mathrm{MF})}(t) & :=\mathbf{G}^{(\mathrm{MF})}(t) * \widetilde{\mathbf{H}}(t) \\
& =\left[\begin{array}{cccc}
R_{1}(t) & R_{1,2}(t) & \cdots & R_{1, K}(t) \\
R_{2,1}(t) & R_{2}(t) & \cdots & R_{2, K}(t) \\
\vdots & \vdots & \ddots & \vdots \\
R_{K, 1}(t) & R_{K, 2}(t) & \cdots & R_{K}(t)
\end{array}\right]
\end{aligned}
$$

Denoting the discrete-time version of $h_{m k}\left(T_{d}-t\right)=$ $\sum_{l=0}^{L} \alpha_{m k, L-l} \delta\left(t-l T_{c}\right)$ as an $(L+1)$ vector $\overline{\mathbf{h}}_{m k}:=$ $\left[\begin{array}{llll}\alpha_{m k, L} & \cdots & \alpha_{m k, 1} & \alpha_{m k, 0}\end{array}\right]^{T}$, then the discrete-time counterparts of $R_{m k}(t), R_{m j, m k}(t), R_{k}(t), R_{j, k}(t)$, respectively, can be formulated as

$$
\begin{aligned}
\mathbf{R}_{m k} & =\eta_{k m} \overline{\mathbf{h}}_{m k} * \mathbf{h}_{m k} \\
& =\eta_{k m}\left[\begin{array}{ccccc}
\alpha_{m k, L} & 0 & \cdots & \cdots & 0 \\
\vdots & \alpha_{m k, L} & \ddots & \ddots & \vdots \\
\alpha_{m k, 0} & \vdots & \ddots & \ddots & \vdots \\
0 & \alpha_{m k, 0} & \ddots & \ddots & 0 \\
\vdots & 0 & \ddots & \ddots & \alpha_{m k, L} \\
\vdots & \vdots & \ddots & \ddots & \vdots \\
0 & 0 & \cdots & 0 & \alpha_{m k, 0}
\end{array}\right] \mathbf{h}_{m k} \\
& =\eta_{k m} \overline{\mathbf{H}}_{m k} \mathbf{h}_{m k}, \\
\mathbf{R}_{m j, m k} & =\eta_{j m} \overline{\mathbf{h}}_{m j} * \mathbf{h}_{m k}=\eta_{j m} \overline{\mathbf{H}}_{m j} \mathbf{h}_{m k}, \quad \forall j \neq k,
\end{aligned}
$$


where $\overline{\mathbf{H}}_{m k}, \overline{\mathbf{H}}_{m j}$ are $(2 L+1) \times(L+1)$ matrices. $\mathbf{R}_{m k}, \mathbf{R}_{m j, m k}, \mathbf{R}_{k}, \mathbf{R}_{j, k}$ all are vectors with size $(2 L+1) \times 1$. Therefore, the magnitude of $\overline{\mathbf{h}}_{m k} * \mathbf{h}_{m k}$ will coherently add up at the central $\left((L+1)\right.$ th) position of $\mathbf{R}_{m k}$, in which the magnitude of the peak is $\mathbf{R}_{m k}(L+1)=\eta_{k m} \sum_{l=0}^{L} \alpha_{m k, l}^{2}=$ $\left\|\mathbf{h}_{m k}\right\|^{2} / \sqrt{M}\left\|\mathbf{h}_{m k}\right\|=\left\|\mathbf{h}_{m k}\right\| / \sqrt{M}$. On the other hand, the other terms of $\overline{\mathbf{h}}_{m j} * \mathbf{h}_{m k}$ will add up noncoherently and symmetrically distributed about $\mathbf{R}_{m k}(L+1)$ :

$$
\begin{gathered}
\mathbf{R}_{k}=\sum_{m=1}^{M} \mathbf{R}_{m k}=\left[\begin{array}{llll}
\mathbf{R}_{1 k} & \mathbf{R}_{2 k} & \cdots & \mathbf{R}_{M k}
\end{array}\right] \mathbf{1}_{M}, \\
\mathbf{R}_{j, k}=\sum_{m=1}^{M} \mathbf{R}_{m j, m k}=\left[\begin{array}{llll}
\mathbf{R}_{1 j, 1 k} & \mathbf{R}_{2 j, 2 k} & \cdots & \mathbf{R}_{M j, M k}
\end{array}\right] \mathbf{1}_{M} .
\end{gathered}
$$

It follows that the peak of $\mathbf{R}_{k}=\sum_{m=1}^{M} \mathbf{R}_{m k}$ is at $\mathbf{R}_{k}(L+1)$ with the magnitude further be enhanced as $\mathbf{R}_{k}(L+1)=$ $1 / \sqrt{M} \sum_{m=1}^{M}\left\|\mathbf{h}_{m k}\right\|$.

The samples of CMF output within the $i$ th bit interval at the $k$ th RT can be expressed as

$$
\mathbf{r}_{k}(i)=a_{k} d_{k}(i) \tilde{\mathbf{c}}_{k}+\sum_{\substack{j=1 \\ j \neq k}}^{K} a_{j} d_{j}(i) \tilde{\mathbf{c}}_{j k}+\mathbf{n}_{k}(i), \quad k=1, \ldots, K,
$$

where $\tilde{\mathbf{c}}_{k}$ represents the effective signature vector of the $k$ th user. It arises from the CMF output's chip-rate samples within a bit of the composite waveform, $c_{k}(t) * R_{k}(t)$. It is evident that $\tilde{\mathbf{c}}_{k}$ can be formulated as

$$
\tilde{\mathbf{c}}_{k}=\left[\begin{array}{llll}
\mathbf{c}_{k} & \mathbf{c}_{k, 1} & \cdots & \mathbf{c}_{k, 2 L}
\end{array}\right] \mathbf{R}_{k}=\mathbf{C}_{k} \mathbf{R}_{k}
$$

where each $\mathbf{c}_{k, l}$ stands for an $l$-chips delayed version of the $k$ th user's TH code. $C_{k}$ is an $N_{f} N_{c} \times(2 L+1)$ matrix. Similarly, we may formulate the $j$ th $(j \neq k)$ user's effective signature vector at the $k$ th $\mathrm{RT}$ as

$$
\tilde{\mathbf{c}}_{j k}=\left[\begin{array}{llll}
\mathbf{c}_{j} & \mathbf{c}_{j, 1} & \cdots & \mathbf{c}_{j, 2 L}
\end{array}\right] \mathbf{R}_{j, k}=\mathbf{C}_{j} \mathbf{R}_{j, k} .
$$

Since the peak of $\mathbf{R}_{k}$ is at $\mathbf{R}_{k}(L+1)$, thereby, after convolving with $c_{k}(t)$, this will shift the positions of the desired user's TH pulse train in $c_{k}(t)$ by $(L+1)$ chips. Therefore, to capture the energy of the desired user, we propose to design a simple correlation receiver to extract the energies at the positions of these peak components. Therefore, the weight vector of the proposed correlation receiver should be chosen as $\mathbf{c}_{k, L}$. The output of the correlation receiver can be obtained as

$$
\begin{aligned}
z_{k}^{(\mathrm{MF})}(i)= & \mathbf{c}_{k, L}^{T} \mathbf{r}_{k}(i) \\
= & \mathbf{R}_{k}(L+1)\left(N_{f}-1\right) a_{k} d_{k}(i) \\
& +\mathbf{c}_{k, L}^{T} \sum_{\substack{j=1 \\
j \neq k}}^{K} a_{j} d_{j}(i) \tilde{\mathbf{c}}_{j k}+\mathbf{c}_{k, L}^{T} \mathbf{n}_{k}(i) .
\end{aligned}
$$

Upon defining $\beta_{j}:=\mathbf{c}_{k, L}^{T} \widetilde{\mathbf{c}}_{j k}$, then the averaged SINR at the output of the $k$ th user's correlation receiver can be obtained as

$$
\begin{aligned}
& \gamma_{k}^{(\mathrm{MF})}=\frac{\left(\mathbf{R}_{k}(L+1)\right)^{2} a_{k}^{2}\left(N_{f}-1\right)^{2}}{\sum_{j=1, j \neq k}^{K} a_{j}^{2}\left|\beta_{j}\right|^{2}+\sigma^{2}\left(N_{f}-1\right)}, \\
& P E_{k}^{(\mathrm{MF})}=2^{1-K} \sum_{\substack{d_{1} \ldots d_{K} \in\{-1,+1\} \\
d_{k}=1}} Q \\
& \cdot\left(\frac{\mathbf{R}_{k}(L+1)\left(N_{f}-1\right) a_{k}+\sum_{j=1, j \neq k}^{K} \beta_{j} a_{j} d_{j}}{\sigma \sqrt{N_{f}-1}}\right), \\
& k=1, \ldots, K
\end{aligned}
$$

\section{Performance Evaluation}

It is worthy to note that channel reciprocity is essential for using the prefiltering technique. Throughout the paper, we assume that channels are reciprocal between the AP and each RT; thereby, the MISO channel coefficients can be estimated by the AP by receiving sounding pulses (the sounding pulse should be made short enough to approach $\delta(t))$ from each of the RT. Therefore, the transmitter has full knowledge of (or can perfectly estimate) the MISO channel's information.

We first assume the average power of the path with index $l=0$ to be normalized to be unity, that is, $\Omega_{0}=1$. The log-normal fading amplitude $\xi_{l}$ is generated by $\xi_{l}=\exp \left(\kappa_{l}\right)$, where $\kappa_{l}$ is a Gaussian random variable, $\kappa_{l} \sim N\left(\mu_{l}, \sigma_{l}^{2}\right)$. To satisfy the second moment of the log-normal random variable [24], $E\left\{\xi_{l}^{2}\right\}=\exp \left(2\left(\mu_{l}+\sigma_{l}^{2}\right)\right)=\Omega_{0} \exp (-\rho l)$, we have $\mu_{l}=-\sigma_{l}^{2}-(\rho l / 2)$. We apply $\rho=0.1, \sigma_{l}^{2}=1$ in all simulation examples (i.e., $\left.\kappa_{l} \sim N(-1-(l / 20), 1)\right)$. For a fixed $L$, we generate 100 sets of channel parameters, $\left\{\alpha_{m k, l}\right\}_{l=0}^{L}$. Each data set is employed for simulation, and the result is obtained by taking average of the 100 independent trials.

Without loss of generality, we assume that user 1 is the desired user hereafter. Unless otherwise mentioned, we set the parameters $N_{f}=20, N_{c}=35, L=15, P=$ $20, K=10$, and each user's SNR, which is defined as $\mathrm{SNR}_{k}:=10 \log \left(a_{k} / \sigma\right)^{2}$, is set to be $15 \mathrm{~dB}$ throughout all the simulation examples. Figure 2 presents the averaged SINR $\left(\gamma_{1}\right)$ with respect to the number of transmitting antennas $M$, where the TR MF, LS (as $M<K(L+P) / P$ ), and $\mathrm{ZF}$ (as $M \geq K(L+P) / P$ ) schemes are provided for comparison. It is verified for both the ZF- and LS-based prefilters that system performance improves as $M$ increases (larger transmit diversity), nevertheless, the performance of the TR MF scheme is only slightly improved. This may result from the increase of interference power for larger $M$ in the TR MF scheme. Figure 3 shows both the $\gamma_{1}$ and BER performance with respect to $\mathrm{SNR}_{1}$, where the performance of TR MF $(M=20)$, TR MF $(M=10)$, ZF $(M=20)$, and LS $(M=10)$ are displayed for comparison. As expected, 


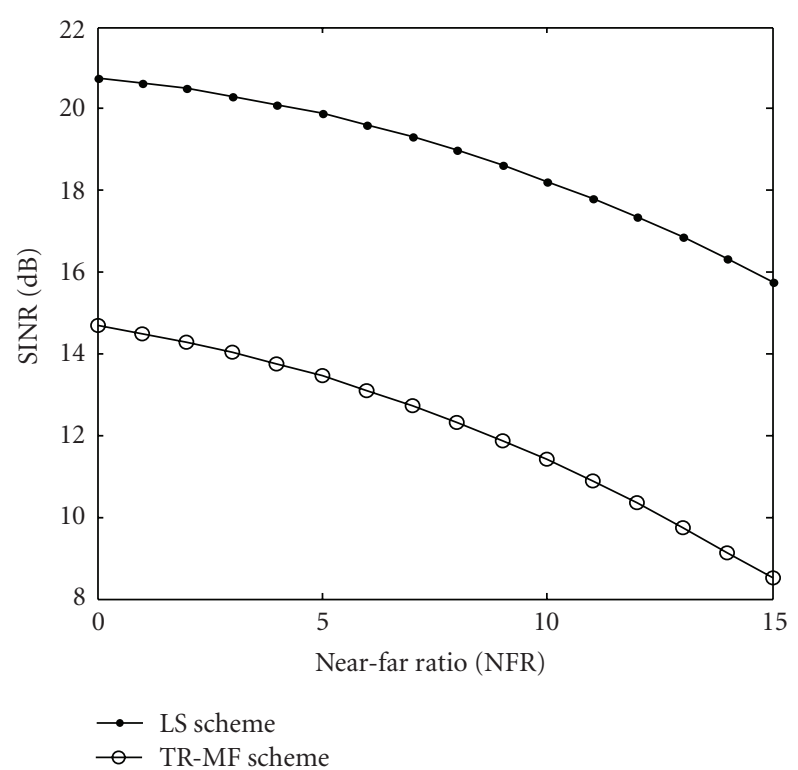

(a)

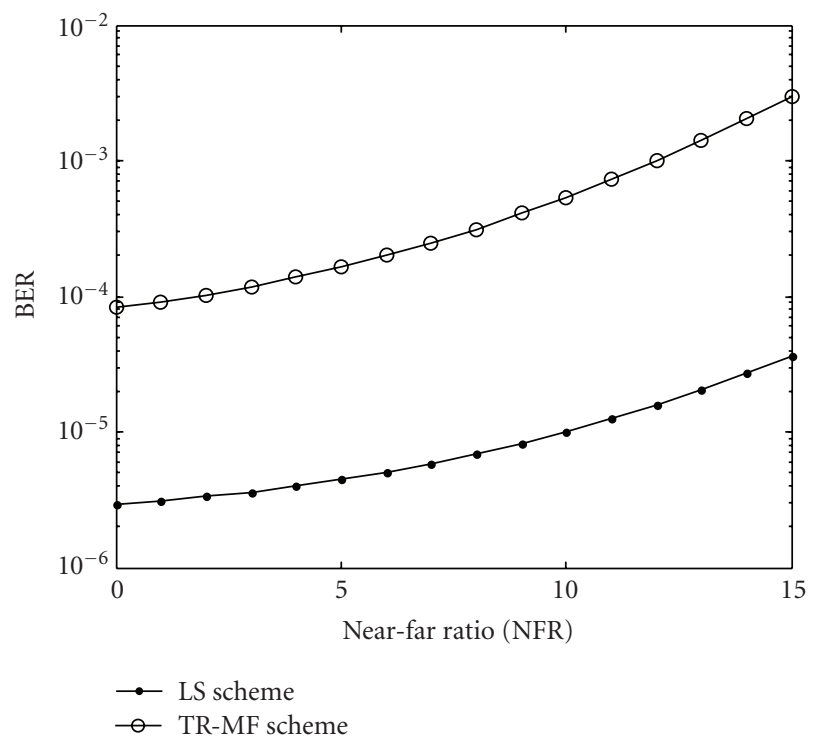

(b)

FIGURE 4: System performance with respect to near-far ratio (NFR) for the TR MF and LS schemes. (a) SINR performance with respect to the near-far ratio (b) BER performance with respect to the near-far ratio.

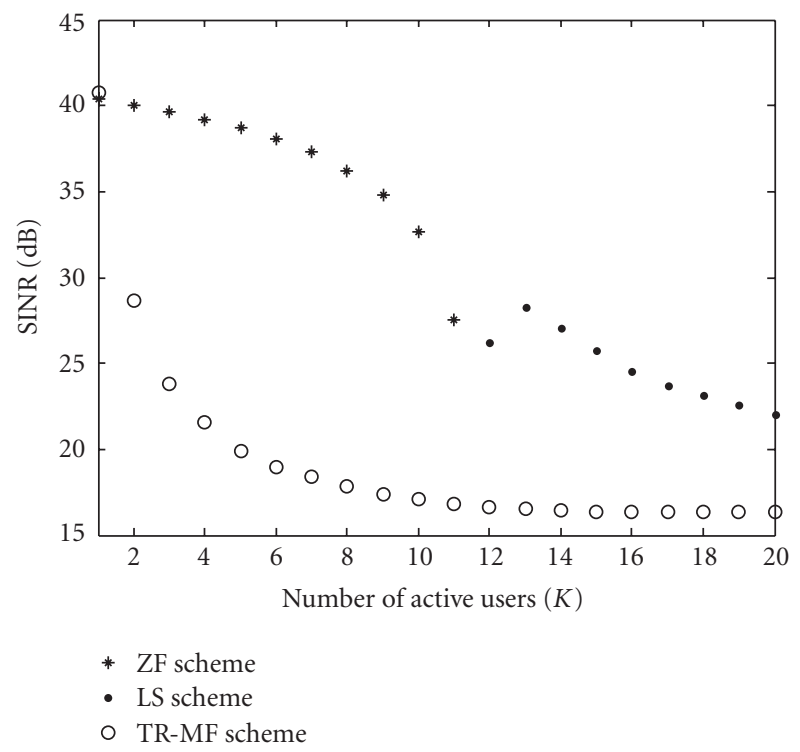

FIGURE 5: SINR performance with respect to the number of active users.

$\gamma_{1}$ increases (BER decreases) in accordance with $\mathrm{SNR}_{1}$. The $\mathrm{ZF}$ scheme performs the best among the four curves since MUI has been completely removed. To measure the near-far resistance characteristics of the TR MF and the LS schemes (the ZF scheme is essentially near-far resistant), we first set all but one of the interferers' (e.g., the $k$ th user) amplitudes to be the same as the desired user, $a_{1}=a_{2}=\cdots=$ $a_{k-1}=a_{k+1}=\cdots a_{K}$, and define the near-far ratio (NFR) as the power ratio, $\left(a_{k} / a_{1}\right)^{2}$ (in $\left.\mathrm{dB}\right)$. The performance in terms of $\gamma_{1}$ and BER with respect to NFR is depicted in Figures 4(a) and 4(b), respectively, where we set $M=10$. As we vary NFR from 0 to $15 \mathrm{~dB}, \gamma_{1}$ slowly decays, nevertheless, it is still above $8 \mathrm{~dB}$ when NFR is as large as $15 \mathrm{~dB}$. This demonstrates that both schemes are applicable in practical near-far environment. Figure 5 presents $\gamma_{1}$ with respect to the number of active users, where we set $M=20$. The TR MF, $\mathrm{ZF}$ (as $K \leq M P /(L+P)$ ), and LS (as $K>M P /(L+P)$ ) schemes are provided for comparison. As verified by the simulation results, the proposed ZF and LS based schemes are essentially robust to MUI, whereas the performance of TR MF scheme degrades as $K$ increases. Specifically, $\gamma_{1}$ of both TR MF and ZF schemes coincide at $K=1$ (single user). This is due to the fact that the TR MF scheme is optimum in single-user case. In the final simulation example, we attempt to measure $\gamma_{1}$ of the ZF and the LS schemes with respect to prefilter length, $P$. Let $M=20, L=15$ and $K=10$, thus, LS scheme is implemented as $P<K L /(M-K)=15$, and ZF-based scheme is applied when $P \geq 15$. We can verify from Figure 6 that increasing the temporal diversity effectively enhances system performance.

According to the above results, several remarks can be made.

(1) Though ZF-based scheme outperforms TR-MFbased scheme, nevertheless, the ZF scheme is only applicable when $K(L+P) \leq M P$. For example, if $P=$ $L$, then the number of antenna must be at least twice as large as the number of active users $(K \leq M / 2)$.

(2) It is well known that applying a ZF filter (or equivalently, decorrelating detector) in the receiver to remove MUI will enhance the additive background noise [25]. Whereas, the power normalization factor $\eta_{k}$ dominates system performance of the ZF-based 


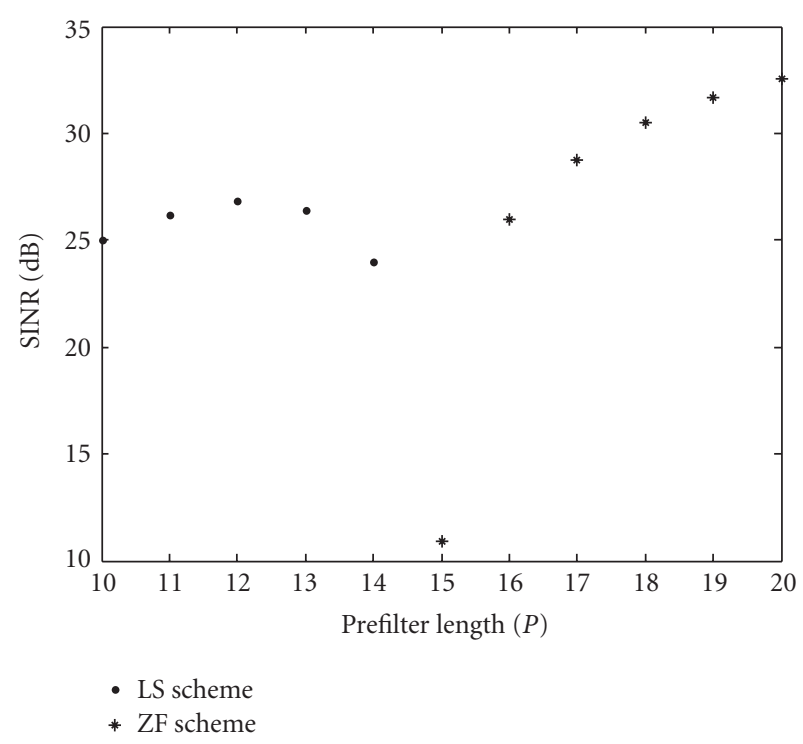

FIGURE 6: SINR performance of the ZF and LS schemes with respect to prefilter length.

prefiltering scheme, a decrease of this leads to performance degradation. As depicted in (18), several factors determine the value of $\eta_{k}$, for example, as $K$ increases, $\eta_{k}$ decreases as well. We have verified in Figure 5 that larger $K$ deteriorates system performance of the ZF-based prefiltering scheme.

\section{Conclusions}

Prefiltering-based multiuser interference suppression techniques have been applied in pulsed UWB system over MISO channel. The benefit of the proposed scheme is that it lessens the burden in signal processing of the RT receiver where a simplified correlation receiver is typically required. The simulation results have demonstrated that the proposed scheme can effectively mitigate near-far problem and suppress MUI. Though binary (antipodal) PAM scheme has been considered in this paper, extension to PPM scheme is without conceptual difficulty.

\section{Acknowledgment}

This research is supported by National Science Council (NSC) of Taiwan under Grant 97-2221-E-212-012.

\section{References}

[1] M. Z. Win and R. A. Scholtz, "Ultra-wide bandwidth timehopping spread-spectrum impulse radio for wireless multipleaccess communications," IEEE Transactions on Communications, vol. 48, no. 4, pp. 679-691, 2000.

[2] M. Z. Win, R. A. Scholtz, and M. A. Barnes, "Ultra-wide bandwidth signal propagation for indoor wireless multiple access communications," in Proceedings of IEEE International Conference on Communications (ICC '97), vol. 1, pp. 56-60, Montreal, Canada, June 1997.
[3] M. Z. Win and R. A. Scholtz, "On the robustness of ultra-wide bandwidth signals in dense multipath environments," IEEE Communications Letters, vol. 2, no. 2, pp. 51-53, 1998.

[4] M. L. Welborn, "System considerations for ultra-wideband wireless networks," in Proceedings of the IEEE Radio and Wireless Conference (RAWCON '01), pp. 5-8, Waltham, Mass, USA, August 2001.

[5] M. Z. Win and R. A. Scholtz, "Impulse radio: how it works," IEEE Communications Letters, vol. 2, no. 2, pp. 36-39, 1998.

[6] N. Boubaker and K. B. Letaief, "Ultra wideband DSSS for multiple access communications using antipodal signaling," in Proceedings of IEEE International Conference on Communications (ICC '03), vol. 3, pp. 2197-2201, Anchorage, Alaska, USA, May 2003.

[7] F. R. Mireles, "Performance of ultrawideband SSMA using time hopping and M-ary PPM," IEEE Journal on Selected Areas in Communications, vol. 19, no. 6, pp. 1186-1196, 2001.

[8] L. Zhao and A. M. Haimovich, "Multiuser capacity of M-ary PPM ultra-wideband communications," in Proceedings of the IEEE Conference on Ultra Wideband Systems and Technologies (UWBST '02), pp. 175-179, Baltimore, Md, USA, May 2002.

[9] R. C. Qiu, "A theory of time-reversed impulse multipleinput multiple-output (MIMO) for ultra-wideband (UWB) communications," in Proceedings of the IEEE International Conference on Ultra-Wideband (ICUWB '06), pp. 587-592, Waltham, Mass, USA, September 2006.

[10] T. Strohmer, M. Emami, J. Hansen, G. Papanicolaou, and A. J. Paulraj, "Application of time-reversal with MMSE equalizer to UWB communications," in Proceedings of IEEE Global Telecommunications Conference (GLOBECOM '04), vol. 5, pp. 3123-3127, Dallas, Tex, USA, November-December 2004.

[11] R. C. Qiu, C. Zhou, J. Q. Zhang, and N. Guo, "Channel reciprocity and time-reversed propagation for ultra-wideband communications," IEEE Antenna and Wireless Propagation Letters, vol. 5, no. 1, pp. 269-273, 2006.

[12] C. Zhou, N. Guo, and R. C. Qiu, "Experimental results on multiple-input single-output (MISO) time reversal for UWB systems in an office environment," in Proceedings of IEEE Military Communications Conference (MILCOM '06), Washington, DC, USA, October 2006.

[13] R. C. Qiu, B. Sadler, and Z. Hu, "Time reversed transmission with chirp signaling for UWB communications and its application in confined metal environments," in Proceedings of the IEEE International Conference on Ultra-Wideband (ICUWB '07), pp. 276-281, Singapore, September 2007.

[14] S. Zhao and H. Liu, "Prerake diversity combining for pulsed UWB systems considering realistic channels with pulse overlapping and narrow-band interference," in Proceedings of the IEEE Global Telecommunications Conference (GLOBECOM '05), pp. 3784-3788, St. Louis, Mo, USA, NovemberDecember 2005.

[15] Z. Tian and G. B. Giannakis, "Data-aided ML timing acquisition in ultra-wideband radios," in Proceedings of the IEEE Conference on Ultra Wideband Systems and Technologies (UWBST'03), pp. 142-146, Reston, Va, USA, November 2003.

[16] L. Yang and G. B. Giannakis, "Low-complexity training for rapid timing acquisition in ultra-wideband communications," in Proceedings of the IEEE Global Telecommunications Conference (GLOBECOM '03), pp. 769-773, San Francisco, Calif, USA, December 2003.

[17] Z. Tian, L. Yang, and G. B. Giannakis, "Non-data-aided timing acquisition of UWB signals using cyclostationarity," in Proceedings of the IEEE International Conference on Acoustics, 
Speech, and Signal Processing (ICASSP '03), vol. 4, pp. 121-124, Hong Kong, April 2003.

[18] L. Yang and G. B. Giannakis, "Blind UWB timing with a dirty template," in Proceedings of the IEEE International Conference on Acoustics, Speech and Signal Processing (ICASSP '04), vol. 4, pp. 509-512, Montreal, Canada, May 2004.

[19] H. T. Nguyen, I. Z. Kovacs, and P. C. F. Eggers, "A time reversal transmission approach for multiuser UWB communications," IEEE Transactions on Antennas and Propagation, vol. 54, no. 11, pp. 3216-3224, 2006.

[20] A. F. Molisch, et al., "A comprehensive standardized model for ultrawideband propagation channels," IEEE Transactions on Antennas and Propagation, vol. 54, no. 11, pp. 3151-3165, 2006.

[21] L. Yang and G. B. Giannakis, "Ultrawideband communications: an idea whose time has come," IEEE Signal Processing Magazine, pp. 26-54, November 2004.

[22] A. F. Molisch, J. R. Foerster, and M. Pendergrass, "Channel models for ultra-wideband personal area networks," IEEE Wireless Communications, vol. 10, no. 6, pp. 14-21, 2003.

[23] J. M. Mendel, Lessons in Estimation Theory for Signal Processing, Communications, and Control, chapter 3, Prentice-Hall, Englewood Cliffs, NJ, USA, 1995.

[24] J. L. Devore, Probability and Statistics for Engineering and the Sciences, Duxbury Thomson Learning, 2000.

[25] S. Verdu, Multiuser Detection, Cambridge University Press, Cambridge, UK, 1998. 